\title{
Phenomenology of transition radiation at radio frequencies from ultrahigh-energy showers
}

\author{
Jaime Alvarez-Muñiz ${ }^{1, \star}$, Pavel Motloch ${ }^{2}$, Paolo Privitera ${ }^{2}$, and Enrique Zas ${ }^{1}$ \\ ${ }^{1}$ Departamento de Física de Partículas \& Instituto Galego de Física de Altas Enerxías, Univ. de Santiago \\ de Compostela, 15782 Santiago de Compostela, Spain \\ ${ }^{2}$ Kavli Institute for Cosmological Physics \& Dept. of Physics, Univ. of Chicago, Chicago, IL 60637, USA
}

\begin{abstract}
We explore transition radiation at $\mathrm{MHz}-\mathrm{GHz}$ frequencies as a possible way to detect ultrahigh-energy (UHE) particles. We have developed a general method to calculate transition radiation that extends the well-known Zas-Halzen-Stanev (ZHS) algorithm. We have applied it to the characterization of the frequency and angular properties of the electric field from high-energy showers crossing the boundary between two media. We discuss the potential of transition radiation for the detection of UHE particles in different situations of experimental interest.
\end{abstract}

\section{Introduction}

The origin of UHE cosmic rays (UHECRs) with energies above $\sim 1 \mathrm{EeV}=10^{18} \mathrm{eV}$ is a long-standing question in astroparticle physics [1]. CRs may interact with matter and radiation at the accelerating source, or along their path towards Earth and produce UHE neutrinos. These travel undeflected through the universe and may provide important clues on the origin of UHECRs. A promising detection technique for UHE neutrinos consists in observing the coherent Askaryan radio signal [2] at $\mathrm{MHz}-\mathrm{GHz}$ frequencies from the neutrino-induced shower in a dense and radio-transparent medium [3]. In this contribution we explore transition radiation (TR) [4] at radio frequencies as a possible way to detect UHE particles. TR is emitted by charged particles when crossing the boundary between two media with different indices of refraction. There are various situations of experimental interest where TR may play a relevant role in detection, for instance Earth-skimming neutrinos inducing a shower inside the Earth's crust that emerges from ground into air, or an UHECR developing a particle shower in the atmosphere and arriving at ground [5]). We have developed a general method to calculate the radiation from a shower crossing the boundary between two media [6] that can be applied to the experimental situations described before as well as many others.

\section{Calculation of the radiation}

Our calculation of radio emission from particle showers crossing the boundary between two media is based on the well-known Zas-Halzen-Stanev (ZHS) algorithm [7]. A shower is modeled as a collection of charged particles whose trajectories are divided in small tracks, along which the particle travels

^e-mail: jaime.alvarezmuniz@gmail.com 
at uniform velocity $\boldsymbol{v}$. From the position and time of the endpoints of these tracks, the frequency spectrum of the electric field from a track is derived using Maxwell's equations [7, 8]:

$$
\boldsymbol{E}(\omega, \boldsymbol{x})=\frac{e \mu_{r}}{2 \pi \epsilon_{0} c^{2}} i \omega \frac{e^{i k R}}{R} e^{i(\omega-\boldsymbol{k} \cdot \boldsymbol{v}) t_{1}} \boldsymbol{v}_{\perp}\left[\frac{e^{i(\omega-\boldsymbol{k} \cdot \boldsymbol{v}) \delta t}-1}{i(\omega-\boldsymbol{k} \cdot \boldsymbol{v})}\right]
$$

where $R$ is the distance from the track to the observer assumed to be large enough for the Fraunhofer regime to be valid. $\boldsymbol{k}$ points in the direction from the track to the observer with $k=\omega / c^{\prime}$, where $\omega$ is the frequency and $c^{\prime}=c / n$ the speed of light in the medium with $n$ the refractive index of the medium. $\boldsymbol{v}_{\perp}=-\hat{\boldsymbol{k}} \times(\hat{\boldsymbol{k}} \times \boldsymbol{v})$ is the component of $\boldsymbol{v}$ in the plane perpendicular direction to $\hat{\boldsymbol{k}}=\boldsymbol{k} /|\boldsymbol{k}| . t_{1}$ and $t_{2}=t_{1}+\delta t$ are the absolute times at which the particle passes through the starting point and endpoint of the track, respectively. $\mu_{r}$ is the relative permeability of the medium. The total electric field at the observer's location is calculated by superposition of the electric field from each track, with relative phase shifts due to different spacetime positions properly taken into account. In the ZHS formula each of the endpoints of the track contributes a term to the electric field. Radiation in a particle trajectory arises from the non-cancellation of the field from adjacent tracks due to their different speeds and orientations w.r.t. the observer. This approach is completely general and it has been succesfully applied to the prediction of Askaryan radiation in particle showers [7] as well as Cherenkov radiation from individual charged particles [8].

In the extended ZHS algorithm (ZHS-TR) for the calculation of radiation from a shower crossing the boundary between a medium 1 and a medium 2, three different types of particle segments are considered (without loss of generality the observer is assumed to be located in medium 2). For tracks fully contained in the first medium, the only contribution comes from the electric field (Eq. 1) emitted into a direction such that when refracted at the boundary it reaches the observer in the second medium (the "transmitted contribution"). For tracks fully contained in the second medium, the "direct contribution" given by Eq. (1) describing the electric field radiated into the observer's direction is calculated, as well as the "reflected contribution" that comes from radiation emitted into a direction such that it is reflected off the boundary into the observer's direction. The appropriate Fresnel coefficients are used for reflection and refraction when necessary. For tracks crossing the border each track is split at the interface into two sub-tracks, as in [9]. Each sub-track is then fully contained in one medium and Eq. (1) can be applied to it. There are thus two endpoints that lie just at the interface. Since they belong to sub-tracks in different media they do not cancel exactly. This partial non-cacellation is the numerical origin of TR. Finally, the electric field produced by the entire shower is obtained from the superposition of the individual contributions of all particle tracks and sub-tracks. The ZHS-TR algorithm has been implemented in the ZHS code for an electromagnetic shower starting in a medium, separated by a planar boundary from a second medium where the observer is located, and all results presented in the following sections correspond to this geometry. If the observer is located in the first medium the roles of the two media must be reversed and two contributions (direct and reflected) are expected from medium 1 and only one (transmitted) from medium 2.

\section{Radiation in showers starting in ice and crossing into air}

In this section we investigate the properties of the radiation emitted by an electromagnetic shower transitioning from homogeneous ice $\left(n_{1}=1.78\right)$ to homogeneous air $\left(n_{2}=1.0003\right)$. The angular distribution of the electric field radiated by a $100 \mathrm{TeV}$ vertical shower simulated with ZHS-TR is shown in Fig. 1. The starting point of the shower in ice was chosen so that the shower maximum occurs approximately at the ice-air boundary. A notable feature is the weak angular dependence of the emitted radiation, when compared to the strongly-beamed coherent Cherenkov radiation [7]. The fluctuations 
around the mean value of the field are due to the fine structure of the shower development in air. We also show in Fig. 1 the contributions to the total electric field (direct, transmitted and reflected). The direct contribution from particle tracks in air is dominant at all angles, and presents a peak at $\sim 1-2^{\circ}$ mainly due to Cherenkov radiation produced in air (Cherenkov angle $\sim 1.4^{\circ}$ ). The reflected contribution is negligible except for the largest angles, where it becomes comparable to the direct contribution as expected. However, the two contributions cancel each other for an observer looking orthogonally to the shower axis, since a $\pi$ phase shift is gained upon reflection. The transmitted contribution from particles in ice is sub-dominant for a vertical shower because the coherent Cherenkov radiation from the Askaryan effect in ice undergoes total internal reflection at the ice-air boundary plane (the Cherenkov angle is larger than the critical angle for $n_{1}=1.78$ ) and cannot reach the observer. This component can become significant for inclined showers, see Figs. 11-14 in [6], when coherent Cherenkov radiation is refracted from ice into air. The frequency spectrum of the radiation is also shown in Fig. 1. For this
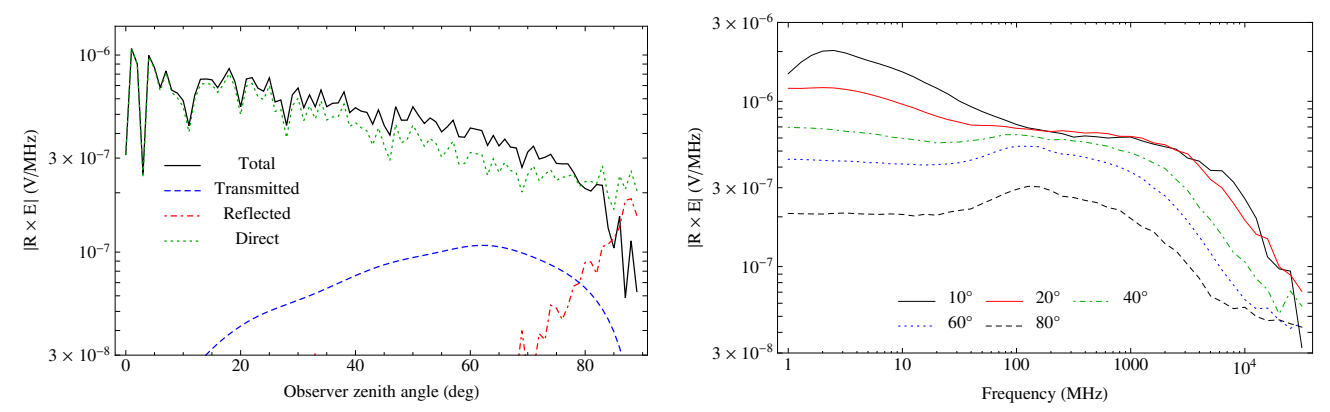

Figure 1. (Left) Angular distribution of the electric field at $1 \mathrm{GHz}$ radiated by a $100 \mathrm{TeV}$ vertical shower crossing from ice to air: total field (black), direct (green short dashes), reflected (red dot-dashes) and transmitted (blue dashes) contributions. The observer is located in air at different angles w.r.t. shower axis. (Right) Frequency spectrum of the electric field radiated by a $100 \mathrm{TeV}$ vertical shower crossing from ice to air, at $10^{\circ}$ (black solid), $20^{\circ}$ (red solid), $40^{\circ}$ (green dot dashes), $60^{\circ}$ (blue short dashes), and $80^{\circ}$ (black long dashes) zenith angle of observation (top to bottom). Each curve is an average of a hundred showers. Figs. taken from [6].

geometry TR is coherent up to $\sim 1 \mathrm{GHz}$ over a wide range of angles, the reason being that showers developing in ice are rather compact in size (Molière radius $\sim 10 \mathrm{~cm}$ ) at the boundary. We have also found that the electric field scales linearly with the charge excess (electrons minus positrons) flowing through the boundary. As a result, its magnitude depends on the stage of evolution of the shower when crossing the boundary [6].

\section{Radiation in showers starting in air and crossing into ice}

We now consider electromagnetic showers transitioning from air into ice. The angular distribution of the electric field radiated by a $10 \mathrm{TeV}$ vertical shower simulated with ZHS-TR is shown in Fig. 2. The observer is located in ice at different angles w.r.t. shower axis and at two azimuthal angles (see caption of Fig. 2). The central peak around $0^{\circ}$ corresponds to the Cherenkov radiation in air refracted into ice that dominates the emission in that angular range, while the two peaks at $\sim \pm 56^{\circ}$ are due to the direct emission of Askaryan radiation in ice peaking at the Cherenkov angle. The frequency spectrum of the radiation is shown in Fig. 2. Showers developing first in air are rather spatially extended in the lateral dimension (Molière radius in air $\sim 1 \mathrm{~km}$ ) and for that reason the electric field increases linearly with frequency up to a few $\mathrm{MHz}$ above which full coherence is lost for observers around $\sim 0^{\circ}$ 

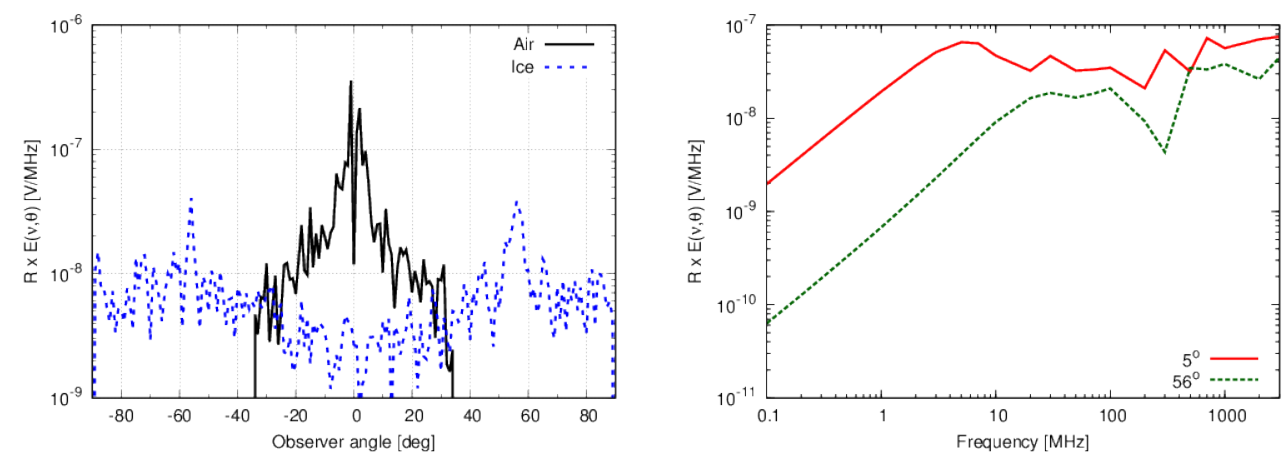

Figure 2. (Left) Angular distribution of the electric field at $1 \mathrm{GHz}$ radiated by a $10 \mathrm{TeV}$ vertical shower crossing from air to ice: total field from the shower in air transmitted into ice (black) and direct+reflected field from the shower in ice (blue dashed). The observer is located in ice at different angles w.r.t. shower axis and at azimuthal angles $0^{\circ}$ (positive values of observer angle) and $+180^{\circ}$ (negative values). (Right) Frequency spectrum of the electric field radiated by a $10 \mathrm{TeV}$ vertical shower crossing from air to ice, at $5^{\circ}$ (red solid) and $56^{\circ}$ (green dashed) observation angles.

where the transmitted radiation dominates the emission (see left panel of Fig. 2). Observers at angles around the Cherenkov angle in ice $\sim 56^{\circ}$ mainly see the direct radiation from the shower in ice, whose spectrum is known to increase linearly with frequency up to a few hundreds of $\mathrm{MHz}$ [7], above which full coherence is lost.

\section{Conclusions}

We have developed a general method based on the ZHS formalism to calculate the electric field radiated by the charged particles in a high-energy shower developing in two different media. Transition radiation naturally arises from this procedure, in addition to the coherent Askaryan radiation produced by the shower particles within each medium. Our so-called ZHS-TR algorithm has general applicability and could be used in various situations of experimental interest. The ZHS-TR algorithm could be also implemented in other shower Monte Carlo codes such as ZHAireS [10] that can handle showers induced by protons, heavier nuclei and neutrinos in the atmosphere or in dense media.

\section{References}

[1] A.A. Watson, Rep. Prog. Phys. 77, 036901 (2014).

[2] G.A. Askar'yan, Soviet Physics JETP 14, 441 (1962); Soviet Phys. JETP 21, 658 (1965).

[3] A. Connolly and A. Vieregg, arXiv:1607.08232v1 [astro-ph.HE].

[4] V. Ginzburg and I. Frank, J. Phys. (USSR) 9, 353 (1945).

[5] K.D. de Vries et al. Astropart. Phys. 74, 96 (2016).

[6] P. Motloch, J. Alvarez-Muñiz, P. Privitera, E. Zas Phys. Rev. D 93, 043010 (2016).

[7] E. Zas, F. Halzen, T. Stanev, Phys. Rev. D 45, 362 (1992).

[8] D. García-Fernández et al., Phys. Rev. D 87, 023003 (2013).

[9] C.W. James, H. Falcke, T. Huege, M. Ludwig, Phys. Rev. E 84, 056602 (2011).

[10] J. Alvarez-Muñiz, W.R. Carvalho Jr., M. Tueros, E. Zas, Astropart. Phys. 35, 287 (2012);

J. Alvarez-Muñiz, W.R. Carvalho, E. Zas, Astropart. Phys. 35, 325 (2012). 